\title{
Progressive Left Ventricular Hypertrophy After Ozaki Procedure: A Case Report
}

\author{
Trang Thi Thu Nguyen, ${ }^{1}$ Anh Tuan Vo, ${ }^{1}$ Danh Vo Tan Nguyen, ${ }^{1}$ Thanh Tri Vu,,${ }^{2,3}$ Dinh Hoang Nguyen ${ }^{1,3}$ \\ ${ }^{1}$ Cardiovascular Surgery Department, University Medical Center of Ho Chi Minh City, Ho Chi Minh City, Viet Nam; ${ }^{2}$ University \\ Medical Center of Ho Chi Minh City - Branch 2, Ho Chi Minh City, Viet Nam; ${ }^{3}$ Cardiothoracic and Vascular Surgery Department, \\ University of Medicine and Pharmacy at Ho Chi Minh, Ho Chi Minh City, Viet Nam
}

\section{ABSTRACT}

We describe a 57-year-old man with symptomatic severe aortic stenosis who underwent aortic valve reconstruction with glutaraldehyde-treated autologous pericardium with the Ozaki technique (Ozaki procedure). Seven months later, he rapidly developed progressive left ventricular hypertrophy with a left ventricular outflow tract obstruction. This required a reoperation for septal myectomy.

\section{INTRODUCTION}

Asymmetric septal hypertrophy (ASH) is typically found in hypertrophic obstructive cardiomyopathy (HOCM), but it also could be seen in aortic stenosis (AS) as a result of high afterload in some instances [Authors/Task Force 2014; Dweck 2012; Hess 1983; Tuseth 2010]. It is challenging to diagnose concomitant HOCM in an AS patient with ASH. Sometimes, the left ventricular outflow tract obstruction (LVOTO) could preoperatively be overlooked. We report a case of AS with $\mathrm{ASH}$, and this patient underwent aortic valve reconstruction. He later developed a symptomatic LVOTO that required reoperation for septal myectomy.

\section{CASE REPORT}

A 57-year-old man was admitted to our hospital with a complaint of chest pain on exertion that lasted for 5 months. His diagnosis was severe AS.

On preoperative transthoracic echocardiography (TTE), we noticed an aliasing of flow in the LVOT, unclear systolic anterior motion (SAM). (Figure 1) (Figure 2) On computed tomography, no significant LVOTO was recorded. The left ventricular wall thickness of each segment is presented in Table 1. The papillary muscles had normal locations (Figure 3).

The patient underwent Ozaki procedure. Intraoperative transesophageal echocardiography (TEE) showed smooth

Received August 1, 2020; accepted August 13, 2020.

Correspondence: Dinh Hoang Nguyen, Cardiovascular Surgery Department, University Medical Center of Ho Chi Minh City, 215 Hong Bang St., Ward 11, District 5, Ho Chi Minh City, Viet Nam; +84-908500090 (e-mail: nguyenhoangdinb@yahoo.com). motion of three new aortic cusps, coaptation length was $9 \mathrm{~mm}$, mild aortic regurgitation, mild SAM and aliasing of flow in LVOT, pressure gradient through the aortic valve and LVOT was $16 \mathrm{mmHg}$. We decided not to perform septal myectomy (Table 1).

The patient did well postoperatively and had regular follow ups at our outpatient clinic. Results of his postoperative TTE are shown in Table 2. Seven months later, he re-experienced dyspnea on exertion, which worsened after one month of medical treatment. A repeat cardiac computed tomography showed significant increase in $\mathrm{LV}$ wall thickness of all segments (Figure 4).

The patient subsequently was diagnosed with HOCM. He underwent a second operation for septal myectomy (Morrow's procedure). Intraoperative exposure of the novel aortic valve showed three soft cusps without restricted

Left ventricular wall thickness of each segment before and after aortic valve reconstruction

\begin{tabular}{lcc}
\hline Cardiac segments & $\begin{array}{c}\text { LV wall thickness in } \\
\text { diastole (mm) (Pre 1st } \\
\text { operation) }\end{array}$ & $\begin{array}{c}\text { LV wall thickness in } \\
\text { diastole (mm) (Pre 2nd } \\
\text { operation) }\end{array}$ \\
\hline Basal anterior & 10.8 & 24.4 \\
Basal anteroseptal & 19.2 & 24 \\
Basal inferoseptal & 19.3 & 19.3 \\
Basal inferior & 9.4 & 11.6 \\
Basal inferolateral & 9.7 & 12 \\
Basal anterolateral & 11.4 & 17.2 \\
Mid anterior & 11.4 & 18.8 \\
Mid anteroseptal & 15.9 & 22.3 \\
Mid inferoseptal & 17.4 & 22.1 \\
Mid inferior & 12.8 & 16.6 \\
Mid inferolateral & 10.4 & 12.6 \\
Mid anterolateral & 10.3 & 13.3 \\
Apical anterior & 8.5 & 16.2 \\
Apical septal & 10.7 & 11.6 \\
Apical inferior & 9.4 & 17.2 \\
Apical lateral & 11.6 & 12.2 \\
Apex & 2 & 5 \\
& &
\end{tabular}




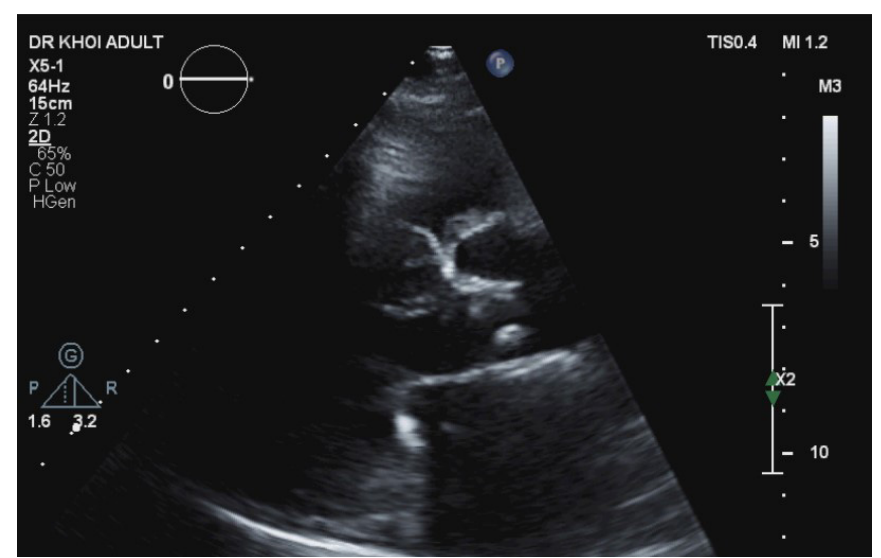

Figure 1. Preoperative echocardiography: Mild SAM.

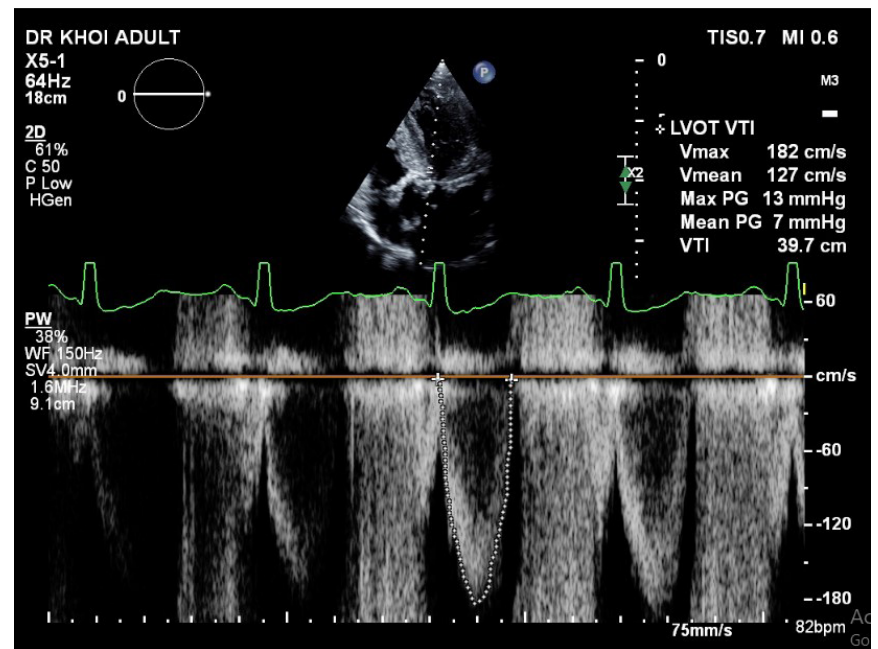

Figure 2. Preoperative echocardiography: Pulse Doppler of flow measured at the level of LVOT.

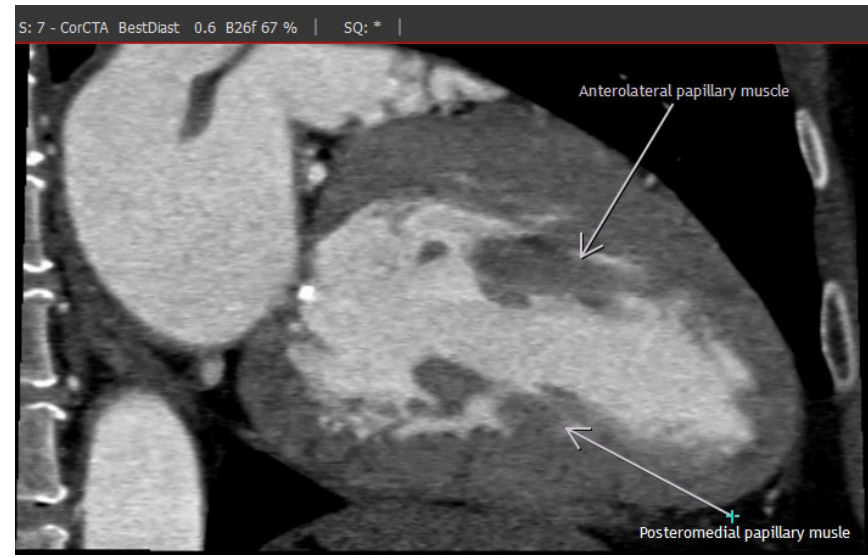

Figure 3. Preoperative cardiac computed tomography before first operation showed normal origins of the papillary muscles.

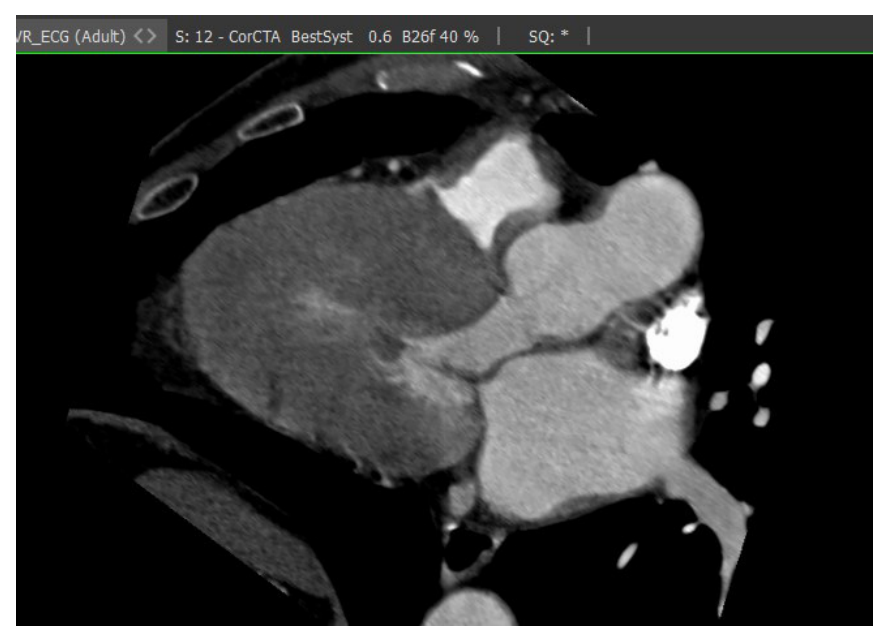

Figure 4. Cardiac computed tomography before the second operation: Significant increase of left ventricular wall diameter and severe SAM were noted.

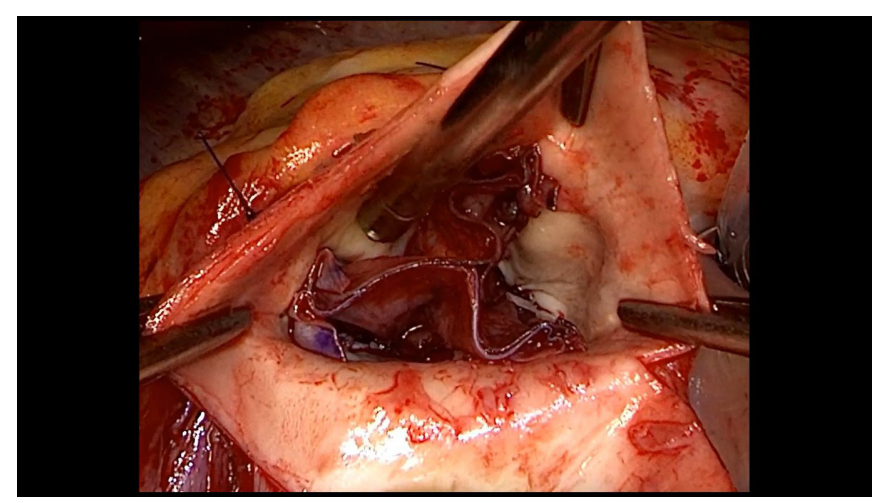

Figure 5. Reconstructed aortic valve.

movement (Figure 5). Histologic results of surgical specimens disclosed hyalinized cardiomyocytes with lymphocytes infiltration, and no typical characteristics of HOCM were recorded on pathology. Thus, the final diagnosis was rapid left ventricular hypertrophy (LVH) after aortic valve reconstruction due to ASH and SAM (Figure 5).

No re-obstruction of LVOT on echocardiography during outpatient follow up was recorded.

\section{DISCUSSION}

ASH is one of the typical signs of HOCM [Authors/Task Force 2014]. However, this entity may be discovered in AS without the presence of HOCM [Dweck 2012; Hess 1983; Tuseth 2010]. In a series of 1,719 asymptomatic AS patients, ASH was detected in $22 \%$ of the patients, independent of the 
severity of AS [Tuseth 2010]. When Hess studied myocardial histologic features of ventricular septum in 24 severe AS cases, ASH was present in $50 \%$ of patients. Hence, no typical morphologic abnormalities of HCM were seen [Hess 1983]. Thus, the presence of ASH in an AS patient does not determine the diagnosis of HOCM.

In ASH patients without LVOTO, septal myectomy is controversial and not recommended as a routine treatment [Authors/Task Force 2014]. In our first operation, the aortic valve was reconstructed while the septum was left untouched. This decision was made based on the following reasons: LVOTO was not clearly diagnosed by the cardiologists, it was believed that the high afterload condition would resolve after reconstruction of the aortic valve, and there was concern of possible complications of septal myectomy.

At the second operation, HOCM was thought to be the cause of SAM and LVOTO. However, the histologic features of septal specimens was not typical of HOCM, which commonly entails myocyte hypertrophy, myocyte disarray, and altered collagen fiber morphology [Hughes 2004]. This excluded the diagnosis of HOCM in our patient. To explain this, we assumed the negative effect of ASH and SAM exposed after aortic valve reconstruction. Normally, in AS patient with severe LVOTO, the latter would reveal in the early postoperative phase [Bartunek 1996; Routledge 2005]. In our case, seven months postoperatively, most of the LV segments had been thickened, and LVOTO had become significant. We thought the LVOTO caused by ASH and SAM was not severe at the time of the aortic valve surgery. However, the LVOT pressure gradient was enough to cause progressive LVH after a period of time. This subsequently led to a diminution of LV volume, as a consequence, SAM and LVOTO increased, thus creating a vicious circle. This "unmasked" dynamic subaortic obstruction and progressive LVH phenomenon was also proven by Panza et al: The relief of the stenosis aortic valve would expose the high-velocity flow through the LVOT, thereby enhancing SAM and obstruction degree, creating a pathological circle [Panza 1988]. During aortic valve surgery for aortic stenosis, we recommend considering septal myectomy in patients who have preoperative ASH and SAM. Intraoperative TEE is necessary to discover SAM and LVOTO which preoperatively could be overlooked.

\section{REFERENCES}

Authors/Task Force, m., et al. 2014. 2014 ESC Guidelines on diagnosis and management of hypertrophic cardiomyopathy: the Task Force for the Diagnosis and Management of Hypertrophic Cardiomyopathy of the European Society of Cardiology (ESC). Eur Heart J. 35(39): p. 2733-79.

Bartunek J, et al. 1996. Abnormal systolic intraventricular flow velocities after valve replacement for aortic stenosis. Mechanisms, predictive factors, and prognostic significance. Circulation. 93(4): p. 712-9.

Dweck MR, et al. 2012. Left ventricular remodeling and hypertrophy in patients with aortic stenosis: insights from cardiovascular magnetic resonance. J Cardiovasc Magn Reson. 14: p. 50.

Hess OM, et al. 1983. Asymmetric septal hypertrophy in patients with aortic stenosis: an adaptive mechanism or a coexistence of hypertrophic cardiomyopathy? J Am Coll Cardiol. 1(3): p. 783-9.

Hughes SE. 2004. The pathology of hypertrophic cardiomyopathy. Histopathology. 44(5): p. 412-27.

Panza JA and Maron BJ. 1988. Valvular aortic stenosis and asymmetric septal hypertrophy: diagnostic considerations and clinical and therapeutic implications. Eur Heart J. 9 Suppl E: p. 71-6.

Routledge T and Nashef SA. 2005. Severe mitral systolic anterior motion complicating aortic valve replacement. Interact Cardiovasc Thorac Surg. 4(5): p. 486-7.

Tuseth N, et al. 2010. Asymmetric septal hypertrophy - a marker of hypertension in aortic stenosis (a SEAS substudy). Blood Press. 19(3): p. $140-4$. 